\title{
Aprovechamiento de una planta astilladora en la Décima Región (Chile)
}

\author{
Yield of a chipping plant in the X Region (Chile) \\ VICTOR SANDOVAL V ${ }^{1}$, LUIS INZUNZA D. ${ }^{2}$ \\ ${ }^{1}$ Instituto de Manejo Forestal, Universidad Austral de Chile, Casilla 567, Valdivia, Chile. \\ E-mail: vsandova@uach.cl \\ 2 Instituto de Tecnología de Productos Forestales, Universidad Austral de Chile, Casilla 567, Valdivia, Chile. \\ E-mail: linzunza@uach.cl

\section{SUMMARY} \\ The yield of a standard chipping plant that operates with roundwood (logs) obtained from managed natural forests \\ in the Tenth Region (Chile) was studied. The results indicated a productivity of $93.6 \%$ for $\log$ from 1.00 to \\ 1.22 m length and less than $40 \mathrm{~cm}$ in diameter, $92.7 \%$ for logs of $2.44 \mathrm{~m}$ in length and less than $40 \mathrm{~cm}$ in \\ diameter, and $85.3 \%$ for pre-cut $\operatorname{logs}$ or $\operatorname{logs}$ of $2.44 \mathrm{~m}$ length and greater than $40 \mathrm{~cm}$; in diameter. The main \\ volume losses related to the roundwood admitted into the plant corresponded to the peeling process, which was \\ followed by losses in the log dimensioning process when they were greater than $40 \mathrm{~cm}$ in diameter.
}

Key words: chips, roundwood, natural forest.

\section{RESUMEN}

Se estudió el aprovechamiento de madera rolliza en una planta astilladora estándar que opera con madera proveniente del manejo de bosques naturales de la Décima Región (Chile). Los resultados indican un aprovechamiento de 93,6\% para rollizos de 1 a 1,22 $\mathrm{m}$ de largo y de diámetro inferior a $40 \mathrm{~cm}, 92,7 \%$ para rollizos de 2,44 $\mathrm{m}$ de largo y diámetro inferior a $40 \mathrm{~cm}$, y un $85,3 \%$ para rollizos previamente partidos o de $2,44 \mathrm{~m}$ de largo y diámetro superior a $40 \mathrm{~cm}$. Las principales pérdidas de volumen con relación a la madera rolliza ingresada a la planta corresponden al proceso de descortezado, seguido de pérdidas en el proceso de dimensionamiento de rollizos de más de $40 \mathrm{~cm}$ de diámetro.

Palabras clave: astillas, madera rolliza, bosques naturales.

\section{INTRODUCCION}

En el sector forestal chileno existen variadas actividades productivas, entre las cuales figura la producción de astillas para la industria de la celulosa con madera procedente de especies naturales. El producto derivado de esta actividad es destinado en alto porcentaje al mercado de exportación, principalmente al continente asiático $\mathrm{y}$, en particular, el mercado japonés (1). El aprovechamien- to en este proceso productivo es todavía algo desconocido y depende principalmente de las dimensiones, del tipo de especies y de rollizos que ingresan a la cadena de producción. En este contexto se procedió a analizar el aprovechamiento de una industria astilladora estándar de la Décima Región (Chile).

El objetivo principal fue determinar estándares de transformación y tasas de pérdida y aprovechamiento de una planta astilladora, que operará 
BOSQUE 25(3): 99-105, 2004

Aprovechamiento de una planta astilladora en la Décima Región (Chile)

con materia prima proveniente de bosques naturales de la Región de Los Lagos.

\section{MATERIAL Y METODOS}

Para alcanzar el objetivo planteado se procedió a estudiar el flujo de producción, desde el ingreso de la madera en fábrica hasta la salida del producto final al término del proceso productivo. En esta etapa se identificaron los puntos de posibles pérdidas de materia prima en el flujo productivo y posteriormente se realizó una cuantificación de dichas pérdidas. El estudio se llevó a cabo separando el muestreo por tipo de materia prima que ingresa a la industria.

En el flujo de producción que presenta la figura 1 se aprecian las distintas etapas y nodos de pérdidas por las cuales pasa la madera hasta ser transformada en astillas.

En el flujo de producción indicado esquemáticamente en la figura 1 , se detectaron los siguientes nodos de pérdida de materia prima:

a) Pérdida por partición de madera sobredimensionada (A).

b) Pérdida por descortezado (B).

c) Pérdidas en sector de rodillos aceleradores o "Buzón" (C).

d) Pérdidas de material fino (D).

Para determinar el aprovechamiento de la planta y calcular los distintos factores de conversión, se diseñó un sistema de muestreo para cuantificar las pérdidas en los distintos nodos identificados y verificar el producto al final de la cadena transportadora.

En el diseño de muestreo aplicado se emplearon unidades muestrales de 29 metros ruma (MR), tamaño suficientemente representativo para evaluar las pérdidas identificadas en el flujo de producción.

La materia prima rolliza que ingresó a la planta se clasificó según las siguientes dimensiones:

- (MR-1,22L<40 D) Metro Ruma de 1 a 1,22 m de largo y diámetro inferior a $40 \mathrm{~cm}$.

- (MR-2,44L<40D) Metro Ruma de 2,44 m de largo y diámetro inferior $40 \mathrm{~cm}$.

- (MR-2,44L>40D) Metro Ruma de 2,44 m de largo y diámetro superior a $40 \mathrm{~cm}$.
El número de unidades muestrales medidas por tipo de materia prima se indica en el cuadro 1.

Todas las unidades muestrales indicadas en el cuadro 1 se sometieron, al ingresar a la planta, a pesaje y cubicado con huincha métrica, con objeto de evaluar en forma exacta las pérdidas que ocurren posteriormente en el flujo de producción.

Las pérdidas identificadas en la cinta transportadora de materia prima, como Descortezado, Buzón o sector de rodillos aceleradores y Material Fino, se evaluaron mediante una submuestra que se indica en el cuadro 2 .

\section{RESULTADOS}

Los resultados obtenidos sobre factores de conversión, tasas de aprovechamiento por tipo de materia prima y pérdida en la cadena de producción se presentan en los siguientes puntos de este trabajo.

Factor de conversión de metro ruma a metro cúbico sólido: El cálculo del factor de conversión se realizó sobre un total de $787 \mathrm{MR}$ procesados. $\mathrm{La}$ metodología que se utilizó en este caso es una combinación de cubicación de trozas, mediante la fórmula de Newton y el método RED-400, descrito por Emanuelli $(2,3)$ y que consiste en la determinación de la proporción de espacios vacíos con relación al total de superficies ocupadas por los MR.

Los estándares de conversión por tipo de materia prima resultantes se presentan en el cuadro 3.

Los factores obtenidos, según muestra el cuadro 3, son similares para los tres tipos de madera rolliza ingresada al proceso de producción y comparables a los definidos para pino insigne por $(4,5)$.

Factor de conversión de metro ruma con corteza a metro cúbico estéreo de chips: Para el cálculo de este estándar de conversión se consideró el procesamiento de 787 metros rumas, distribuidos por tipo de rollizo según indica el cuadro 1. El producto final en chips arrojó los resultados que se muestran en el cuadro 4.

Una estimación más apropiada de los factores de conversión volumétrica de metro ruma a metro 


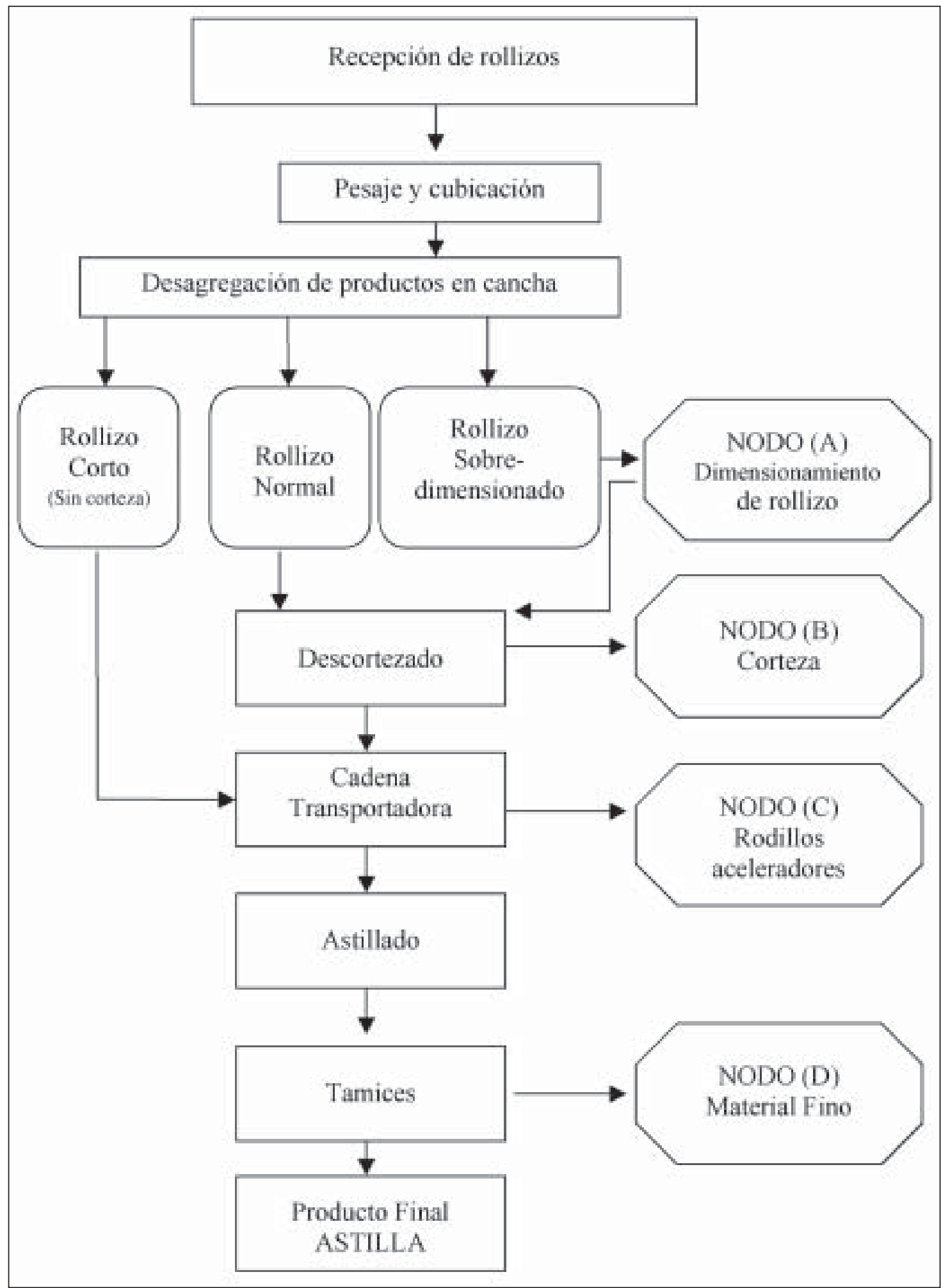

Figura 1. Diagrama de producción en una planta astilladora. Production diagram in a chipping plant. 


\section{CUADRO 1}

Número de unidades muestrales y volumen en metros rumas (MR) procesado en el ensayo.

Number of sampling units and volume (in MR) tested in the analysis.

\begin{tabular}{|lcc|}
\hline Tipo de materia prima & $\begin{array}{c}\mathrm{N}^{\circ} \text { de Unidades } \\
\text { muestrales } \\
\text { (o lotes) }\end{array}$ & $\begin{array}{c}\text { Volumen total procesado } \\
\text { por muestras en } \\
\text { Metros Rumas (MR) }\end{array}$ \\
\hline MR-1,22L<40 D & 8 & 236,00 \\
MR-2,44L<40 D & 8 & 197,32 \\
MR-2,44L>40 D & 13 & 354,51 \\
Total & 29 & 787,83 \\
\hline
\end{tabular}

CUADRO 2

Número de unidades muestrales de material por nodo de pérdida. Number of material sampling units per loss node.

\begin{tabular}{|l|c|c|c|}
\hline \multirow{2}{*}{ Nodo de pérdida } & \multicolumn{3}{|c|}{$\mathrm{N}^{\circ}$ de Unidades muestrales por tipo de materia prima } \\
\cline { 2 - 4 } & $\mathrm{MR}-1,22<40$ & $\mathrm{MR}-2,44<40$ & $\mathrm{MR}, 2,44>40$ \\
\hline Descortezador & 0 & 5 & 8 \\
Buzón & 8 & 4 & 9 \\
Fino & 2 & 2 & 0 \\
\hline
\end{tabular}

CUADRO 3

Factores de conversión de MR a metro cúbico sólido ( $\mathrm{m}^{3}$ s.s.c.). MR to debarked solid cubic meter conversion factors ( $\mathrm{m}^{3}$ s.s.c. $)$.

\begin{tabular}{|l|c|cc|}
\hline Tipo de materia prima & $\begin{array}{c}\text { Factor de Conversión } \\
\text { MR / } \mathrm{m}^{3} \text { s.s.c. }\end{array}$ & \multicolumn{2}{|c|}{ Valores } \\
\cline { 3 - 4 } & 1,735 & 1,401 & 1,894 \\
MR-1,22L<40 D & 1,720 & 1,440 & 1,895 \\
MR-2,44L<40 D & 1,623 & 1,403 & 1,807 \\
MR-2,44L>40 D & \multicolumn{2}{|c}{} \\
\hline
\end{tabular}

cúbico estéreo de chips, es el rendimiento en peso del producto final y sus respectivas pérdidas, el cual se presenta en el siguiente punto.

Rendimiento por tipo de materia prima: Los rendimientos obtenidos por tipo de rollizos en kilos y porcentual se presentan en forma desagregada por tipo de materia prima en el cuadro 5. En él se describe la magnitud total de pérdidas ocurridas en la cadena de producción.

El cuadro 5 indica que el mejor rendimiento corresponde al rollizo de corta dimensión (1,22 m), con un 93,7\% del peso ingresado como materia prima. Las pérdidas mayores se producen especialmente en rollizos de 2,44 metros de largo y diámetros mayores (rollizo MR-2,44>40). 
BOSQUE 25(3): 99-105, 2004 Aprovechamiento de una planta astilladora en la Décima Región (Chile)

\section{CUADRO 4}

Factores de conversión de metro ruma con corteza a metro cúbico estéreo de chips.

Barked MR to stere cubic meters of chips conversion factors.

\begin{tabular}{|l|c|}
\hline $\begin{array}{l}\text { Tipo de } \\
\text { materia prima }\end{array}$ & $\begin{array}{c}\text { Factor de Conversión MR } \\
\mathrm{a} \mathrm{m}^{3} \text { estéreo de chips }\end{array}$ \\
\hline MR-1,22L<40 D & 4,382 \\
MR-2,44L<40 D & 4,334 \\
MR-2,44L>40 D & 4,089 \\
Valor promedio & 4,268 \\
\hline
\end{tabular}

Los valores máximos, mínimos y promedio de aprovechamiento y pérdidas se presentan en el cuadro 6.

Pérdidas de materia prima por nodos de cadena de producción: La información promedio de pérdida por nodo se presenta en el cuadro 7 , desagregada según tipo de materia prima procesada. Estas cifras corresponden a valores porcentuales del peso total de los metros ruma ingresados.

En la cadena de producción, los valores de pérdidas por tipo de materia prima, según el cuadro 7 y figura 2, son bastante dispares por nodo, acentuándose principalmente en el proceso de

\section{CUADRO 5}

Resumen de aprovechamiento y pérdidas por tipo de materia prima. Yield and loss overview for each kind of raw material.

\begin{tabular}{|l|c|c|c|c|c|c|}
\hline \multirow{2}{*}{$\begin{array}{l}\text { Materia } \\
\text { Prima }\end{array}$} & \multirow{2}{*}{$\begin{array}{c}\text { No promedio de } \\
\text { MR por unidad } \\
\text { de muestreo }\end{array}$} & \multirow{2}{*}{$\begin{array}{c}\text { Peso Medio } \\
\text { por unidad } \\
\text { muestral }(\mathrm{kg})\end{array}$} & \multicolumn{2}{|c|}{ Rendimiento } & \multicolumn{2}{|c|}{ Pérdidas } \\
\cline { 5 - 7 } & & & $(\mathrm{kg})$ & $\%$ & $(\mathrm{~kg})$ & $\%$ \\
\hline MR-1,22<40 & 28,5 & $33.433,1$ & $31.320,3$ & 93,68 & $2.112,9$ & 6,32 \\
MR-2,44<40 & 24,7 & $33.021,5$ & $30.627,4$ & 92,75 & $2.394,1$ & 7,25 \\
MR-2,44>40 & 27,3 & $48.524,8$ & $41.425,6$ & 85,37 & $7.099,2$ & 14,63 \\
\hline
\end{tabular}

\section{CUADRO 6}

Pérdidas y aprovechamiento por tipo de materia prima. Yield and loss for each kind of raw material.

\begin{tabular}{|l|l|c|cr|}
\hline \multirow{2}{*}{$\begin{array}{l}\text { Tipo de } \\
\text { materia prima }\end{array}$} & Tipo de Rollizo & $\begin{array}{c}\text { Valor medio } \\
(\%)\end{array}$ & \multicolumn{2}{|c|}{ Valores (\%) } \\
\cline { 3 - 5 } & & Mínimo & Máximo \\
\hline \multirow{2}{*}{ MR-1,22<40 } & Rendimiento & 93,68 & 90,56 & 97,58 \\
& Pérdidas & 6,32 & 2,42 & 9,44 \\
MR-2,44<40 & Rendimiento & 92,75 & 89,63 & 95,68 \\
& Pérdidas & 7,25 & 4,32 & 10,37 \\
MR-2,44>40 & Rendimiento & 85,37 & 81,12 & 87,68 \\
& Pérdidas & 14,63 & 12,32 & 18,88 \\
\hline
\end{tabular}




\section{CUADRO 7}

Pérdidas porcentuales por materia prima por nodo en el flujo de producción. Percentage losses per node in the production flow for each kind of raw material.

\begin{tabular}{|l|c|c|c|c|c|c|}
\hline \multirow{2}{*}{$\begin{array}{l}\text { Categoría } \\
\text { Rollizo }\end{array}$} & \multicolumn{5}{|c|}{ Pérdidas (\%) } & \multirow{2}{*}{ Total } \\
\cline { 2 - 6 } & Partido & Corteza & Buzón & Fino & Otras & \\
\hline MR-1,22<40 & 0 & 0 & 2,53 & 1,83 & 1,96 & 6,32 \\
MR-2,44<40 & 0 & 5,10 & 0,31 & 0,79 & 1,05 & 7,25 \\
MR-2,44>40 & 4,12 & 8,15 & 0,59 & 0,79 & 0,98 & 14,63 \\
Valores medios & 4,12 & 6,62 & 1,14 & 1,14 & 1,33 & \\
\hline
\end{tabular}

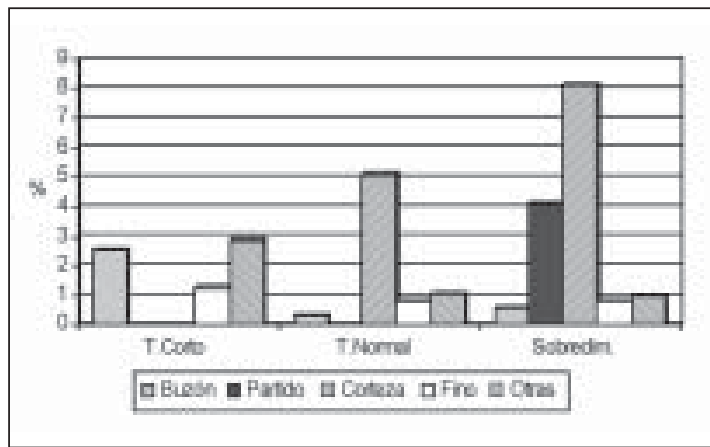

Figura 2. Pérdidas de materia prima según nodo de cadena de producción.

Raw material losses according to nodes in production line.

descortezado. Las pérdidas en el rodillo de aceleración o "buzón" y por material fino son similares.

\section{CONCLUSIONES}

En esta nota técnica se entregan resultados del proceso de transformación de la madera rolliza a astillas. La determinación de estos factores de conversión es elemento necesario para una planificación óptima en el abastecimiento de una planta, como también para efectos de comercialización de esta materia prima.
Los estándares entregados, aunque son de carácter local, y dependen de las características de la madera que ingresa a la industria, permiten distinguir las principales pérdidas en la cadena de producción de una planta típica de astillado.

La principal pérdida, según los resultados, corresponde al proceso de descortezado, el cual representa alrededor de un 5\% del total de la madera ingresada.

Otro factor importante de pérdida es el proceso de partido en rollizos sobredimensionados, el cual contabiliza un $4 \%$. Los restantes factores representan sólo un porcentaje aproximado al $1 \%$ del total.

\section{BIBLIOGRAFIA}

(1) INSTITUTO FORESTAL (CHILE). Boletín Estadístico 61. Santiago, Chile. 1997, p. 28.

(2) EMANUELLI, P., J. CANCINO, F. MILLA. Tres métodos para la determinación de factores de conversión en madera apilada. Agro-ciencia. Concepción, Chile. 1997, p. 27.

(3) EMANUELli, P., J. CANCINO, F. MILlA. Método red-400: Una nueva alternativa para determinar factores de conversión. Agro-ciencia. Concepción, Chile. 1998, p. 13.

(4) PETERS R., M. JOBET, S. AGUIRRE. Compendio de tablas auxiliares para el manejo de plantaciones de pino insigne. Instituto Forestal (Chile), Manual 14. 1985, p. 140.

(5) MOLINARE, V.F. Determinación del volumen sólide contenido en un metro ruma por medio de fotografía terrestre. Tesis Ingeniería Forestal, Universidad de Chile, Santiago, Chile. 1976, p. 61 
BOSQUE 25(3): 99-105, 2004 Aprovechamiento de una planta astilladora en la Décima Región (Chile)

ANEXO

UNIDADES DE COMERCIALIZACIÓN DE ASTILLAS Y FACTORES EMPÍRICOS DE CONVERSIÓN

- Green Metric Ton (GMT): Esta unidad corresponde a una tonelada métrica $(1.000 \mathrm{~kg})$ de astillas verdes.

- Bone Dried Ton (BDT): Esta unidad corresponde a una tonelada de astillas secas ( $0 \%$ contenido de humedad). En esta unidad se deben distinguir las "toneladas largas" (2.240 lbs) (BDTL), las "toneladas cortas" (2.200 lbs) (BDTC) y las toneladas métricas $(1.000 \mathrm{~kg})$

- Bone Dried Metric Ton (BDMT): Esta unidad corresponde a una tonelada métrica (1.000 kg) de astillas secas $(0 \%)$. Estas son las que usualmente se emplean en el comercio mundial de astillas en países con sistema métrico decimal.

- Bone Dry Unit (BDU): Son 2.400 lbs de astillas en condición anhidra. Se emplea en el comercio mundial con países que poseen un sistema de medidas distinto al sistema métrico decimal. En el cuadro A1 se muestran las equivalencias entre los distintos tipos de unidades de comercialización de astillas.

\section{CUADRO A 1}

Conversión entre distintas unidades de peso seco de astillas. Conversion between different units from dry weight of chips.

\begin{tabular}{|l|c|c|c|c|}
\hline Unidades & BDTC & BDTL & BDU & BDMT \\
\hline BDTC & 1,0000 & 1,0182 & 1,0909 & 1,0021 \\
BDTL & 0,9821 & 1,0000 & 1,0714 & 0,9842 \\
BDU & 0,9167 & 0,9333 & 1,0000 & 0,9186 \\
BDMT & 0,9979 & 1,0160 & 1,0886 & 1,0000 \\
\hline
\end{tabular}

La cantidad de madera necesaria, en volumen, para producir una unidad de peso seco de astillas dependerá, esencialmente, de la densidad de la madera que se está astillando. Mientras más densa sea la madera de la especie que se está procesando, menor será el volumen requerido para producir una unidad seca. También influyen el proceso de astillado, el contenido de humedad y la presencia o ausencia de biodeterioro en la madera. De ahí se deriva la complejidad en determinar equivalencias exactas entre toneladas secas y unidades de volumen.

En términos prácticos se debe considerar en madera sana un equivalente para BDMT igual al recíproco de las densidades básicas que varían entre 0,44 y 0,48. Si se asume una producción de finos equivalente a un $5 \%$ se tiene:

$$
(1 / 0,46) \times 1,05=2,3\left(\mathrm{~m}^{3} / \mathrm{BDMT}\right)
$$

Como la madera se comercializa en metros ruma (MR), es importante saber la relación entre MR y metros cúbicos sólido. Esta equivalencia se determina por el factor de apilamiento. En la práctica, se tiene que un MR contiene entre 1,52 y $1,55 \mathrm{~m}^{3}$, donde el factor de apilamiento depende de factores como longitud de las trozas, diámetros, rectitud, etc. Productores estiman que con un MR se pueden producir entre 0,67 y 0,74 BDMT. 


\section{REGLAMENTO DE PUBLICACIONES REVISTA BOSQUE}

BOSQUE acepta trabajos originales relacionados con las Ciencias Forestales en las modalidades de artículos, notas científicas y técnicas, opiniones, reseñas y revisiones bibliográficas, más avances de investigación en castellano e inglés.

ARTÍCULOS. Informan acerca de una investigación inédita de carácter científico, basada en bibliografía actualizada y datos empíricos.

OPINIONES. Analizan, desde un punto de vista personal o con apoyo bibliográfico, un tema de actualidad relacionado con el carácter de la revista, pudiendo originar una controversia. El Editor puede invitar a contribuir con una opinión a un experto en algún tema de interés o de controversia. Se publican máximo dos opiniones por número.

NOTAS TÉCNICAS Y CIENTÍFICAS. Describen metodologías o técnicas nuevas y descubrimientos significativos para el sector forestal.

RESENAAS Y REVISIONES BIBLIOGRÁFICAS. Analizan y critican una publicación de actualidad o un tema específico.

AVANCES DE INVESTIGACION. Informan acerca de investigaciones en desarrollo, con resultados preliminares.

\section{Formato}

Trabajo escrito: impreso tamaño carta $(28 \times 21 \mathrm{~cm})$, márgenes de $2,0 \mathrm{~cm}$ por lado, interlineado a espacio y medio, letra Times New Roman, tamaño 12, con numeración de página en el extremo inferior derecho. La extensión máxima (incluidos cuadros y figuras) será de 30 páginas para los artículos y 18 para las otras contribuciones.

El título principal se presentará en letras bajas y negritas, a diferencia de los títulos de secciones que se escribirán en letras altas. En este título deberá omitirse la mención de los autores de nombres científicos que, sin embargo, sí se presentarán la primera vez que se mencionen en el texto.

Las ecuaciones se escribirán en letra cursiva y se numerarán en el margen derecho con paréntesis cuadrados [ ], luego, en el texto, se mencionarán de acuerdo a esta numeración.
Las unidades de medida utilizadas en el texto deberán circunscribirse al Sistema Internacional (SI), siempre que exista el equivalente.

En la notación numérica, los decimales deberán ser separados por coma (,) y los miles por punto (.).

Las citas bibliográficas en el texto serán del tipo numérico, por orden de aparición, entre paréntesis redondos (): ejemplo: (25); cuando se agrupen, deberán separarse con comas, ejemplo: $(12,25,32)$. Al utilizar vocativos, la cita deberá asociárseles inmediatamente después, ejemplo: Según Gutiérrez (5) y Burschel et al. (16).

Figuras, fotografías y cuadros: el espacio total que éstas ocupen en el trabajo deberá ser menor al 50\% de todo el impreso. Deben incluir un título numerado en forma consecutiva, autoexplicativo en castellano e inglés. En el caso de figuras y fotografías el título va en el margen inferior y en el caso de cuadros en el margen superior. Todos con alta resolución tal, que permitan ser reducidos sin perder legibilidad. Sólo se trabaja en blanco, negro y tonos de grises.

Dentro del texto se debe indicar claramente la ubicación de figuras, fotografías y cuadros, que deben ser agregados al final del texto y grabados en archivos separados.

Bibliografía: se regirá según Norma ISO 690 e ISO 690-2 (referencia de documentos electrónicos):

\section{Libro}

(12) SNEDECOR, G., W. COCHRAN. Métodos estadísticos. $8^{\mathrm{a}}$ ed, México D.F.: Continental. 1981. 703 p.

\section{Capítulo de Libro}

Libro y capítulo son del mismo autor

(4) PARKER, T.J., W.D. HASWELL. A text-book of zoology. $5^{\text {th }}$ ed, vol. 1, London: Macmillan. 1930. Section 12, Phylum Mollusca, p. 663-782.

\section{Libro y capítulo son de autores diferentes}

(6) WRIGLEY, E.A. Parish registers and the historian. In: STEEL, D.J. National index of parish registers. London: Society of Genealogists, 1968, vol. 3, p. 167. 


\section{Publicación Seriada}

(2) TRONCOSO T. J., R. GARRIDO H., X. IBACACHE J. Modelos de localización de instalaciones: una aplicación para la producción y logística forestal. Bosque (Chile), 2002, vol. 23, No 2, p. 57-67.

\section{Patentes}

(17) CARL ZEISS JENA, VEB. Ariordnung zur lichtelektrischen Erfassung der Mitte eines Lichtfeldes. Schweiz: Patentschrift, 608-626. 1979-Jan-15.

\section{Internet}

Básicamente se deben respetar las mismas reglas que para las obras impresas, sin embargo, deberán precisarse el año de publicación, el mes y día, si aparece además de la fecha de consulta:

(3) DUNBAR, B. The 16 Most Frequently Asked Questions about NASA. In: The NASA Homepage. 29 marzo 1996. [citado 14 agosto 1996]. Disponible en: <http://www.nasa.gov/hqpao/Top10.html>.

\section{Estructura de artículos}

La organización de los trabajos deben seguir la siguiente estructura y orden:

Título en castellano ( $\mathrm{Si}$ se desea dejar constancia de financiamiento con el cual contó el trabajo, debe colocar un asterisco al final del título e incluir el nombre a pie de la primera página).

Título en inglés.

Identificación de autores: nombre completo de todos los autores en mayúsculas, dirección de autores (indicando con superíndices en sus nombres direcciones distintas), e-mail.

Summary: debe ser igual al resumen en castellano y con un máximo de 200 palabras.

Key words: idénticas a las palabras claves en castellano. Resumen: debe contener el objetivo, parte de metodología, resultados y conclusiones más relevantes, sin citas bibliográficas.

Palabras claves: como máximo cinco palabras que identifiquen claramente el tema del trabajo.

Introducción: una breve reseña general del tema a tratar, apoyada en bibliografía actual.

Material y métodos: deben quedar claramente indicados la procedencia y número de muestras, análisis estadístico y pruebas a aplicar durante la ejecución del trabajo. Resultados y discusión: entrega de resultados en forma escrita, ayudados de cuadros y figuras, conjuntamente con la discusión y análisis de los datos.
Conclusiones: que se deriven directamente de lo entregado por el trabajo en forma precisa y concisa.

Agradecimientos: dedicados a personas o instituciones (aquí no se debe hacer referencia al financiamiento del trabajo).

Bibliografía: deben indicarse todas las citas del texto en orden numérico y en el formato que se indica.

Anexos: incluye el material extra que se requiera para mayor entendimiento del trabajo expuesto, debidamente mencionados en el texto.

\section{Proceso de publicación}

Recibido el trabajo por triplicado en papel más el disquete en Oficina de BOSQUE (Casilla 567, Valdivia, Chile), el autor principal recibirá una carta de acuse de recibo del Editor.

A continuación el trabajo es analizado por el Comité Editorial y se le destinan los árbitros del Comité Científico. Recibidas las observaciones por escrito de los árbitros, se analiza entre ambos comités la decisión de publicación y/o modificaciones.

El Editor envía a los autores copia de las observaciones Los autores en no más de tres semanas deben devolver a la revista una copia impresa modificada junto a sus archivos, para que los comités analicen nuevamente el trabajo.

Una vez aceptado el trabajo, el Editor envía al autor principal una carta de aceptación indicando bajo qué modalidad será publicado (Artículo o Nota) más el volumen y número de aparición de su trabajo.

En caso de existir nuevas observaciones, se reenvían a autor para que las corrija y siga nuevamente el procedimiento.

Un trabajo es rechazado cuando por segunda vez los autores no realizan las modificaciones de fondo sugeridas por los Comités Científico y Editorial, en cuyo caso todo el material es devuelto al autor principal.

Una vez impresa la revista, el autor principal recibirá sin costo 5 apartados de su contribución.

Para cualquier duda se sugiere visitar nuestra página web: www.uach.cl/revistabosque.

A partir de 2003 Revista BOSQUE es parte de SciELOChile www.scielo.cl, donde podrá encontrar la versión electrónica de sus artículos. 